Stability Measurements for Alignment of the NIF Neutron Imaging System Pinhole Array

D. N. Fittinghoff, D. E. Bower, O. B. Drury, J. M. Dzenitis, M. Frank, R. A. Buckles, C. Munson, C. H. Wilde

March 30, 2011 
This document was prepared as an account of work sponsored by an agency of the United States government. Neither the United States government nor Lawrence Livermore National Security, LLC, nor any of their employees makes any warranty, expressed or implied, or assumes any legal liability or responsibility for the accuracy, completeness, or usefulness of any information, apparatus, product, or process disclosed, or represents that its use would not infringe privately owned rights. Reference herein to any specific commercial product, process, or service by trade name, trademark, manufacturer, or otherwise does not necessarily constitute or imply its endorsement, recommendation, or favoring by the United States government or Lawrence Livermore National Security, LLC. The views and opinions of authors expressed herein do not necessarily state or reflect those of the United States government or Lawrence Livermore National Security, LLC, and shall not be used for advertising or product endorsement purposes.

This work performed under the auspices of the U.S. Department of Energy by Lawrence Livermore National Laboratory under Contract DE-AC52-07NA27344. 


\title{
Stability measurements for alignment of the NIF neutron imaging system pinhole array
}

\author{
David N. Fittinghoff, Dan E. Bower, Owen B. Drury, John M. Dzenitis, Matthias Frank \\ Lawrence Livermore National Laboratory, Livermore CA 94550, fittinghoff1@1lnl.gov
}

\author{
Robert A. Buckles \\ National Security Technologies, LLC, Livermore, CA 94550
}

Carter Munson and Carl H. Wilde

Los Alamos National Laboratory, Los Alamos, NM 87545

The alignment system for the National Ignition Facility's neutron imaging system ${ }^{1-5}$ has been commissioned and measurements of the relative stability of the 90-315 DIM, the front and the back of the neutron imaging pinhole array and an exploding pusher target have been made using the 90-135 and the 90-258 opposite port alignment systems. Additionally, a laser beam shot from the neutron-imaging Annex and reflected from a mirror at the back of the pinhole array was used to monitor the pointing of the pinhole. Over a twelve hour period, the relative stability of these parts was found to be within $\pm 18 \mu \mathrm{m}$ rms even when using manual methods for tracking the position of the objects. For highly visible features, use of basic particle tracking techniques found that the front of the pinhole array was stable relative to the $90-135$ opposite port alignment camera to within $\pm 3.4 \mu \mathrm{m}$ rms. Reregistration, however, of the opposite port alignment systems themselves using the target alignment sensor was found to change the expected position of target chamber center by up to $194 \mu \mathrm{m}$. Lawrence Livermore National Laboratory is 
operated by Lawrence Livermore National Security, LLC, for the U.S. Department of Energy, National Nuclear Security Administration under Contract DE-AC52-07NA27344

\section{Introduction}

The neutron imaging system (NIS) at the National Ignition Facility (NIF) is designed to capture a 2-D image of the source distribution of neutrons from imploding inertial confinement fusion capsules. While in the simplest sense the neutron imager is simply a pinhole camera in which an aperture is used to define an image at a plane, since neutrons penetrate materials far more than photons do in optical pinhole cameras, a neutron imager requires significantly thicker pinholes, which drives strict alignment requirements.

In the case of the NIF NIS, the aperture is an array of pinholes in 20 -cm of gold as shown in Figure 1. The triangular pinholes are single-sided tapers, which are created by scribing triangular grooves in gold layers within the assembly, and have 5- $\mu \mathrm{m}$ openings (height of triangle) at the front of the pinhole and 226- $\mu \mathrm{m}$ openings at the back of the pinhole. The mini-penumbral pinholes are $273-\mu \mathrm{m}$ in diameter at the front surface of the array and $437-\mu \mathrm{m}$ in diameter at the rear surface of the pinhole array. The high aspect ratio of these holes combined with issues related to image reconstruction algorithms produces the strict alignment requirements, which are positioning the front of the pinhole to within $\pm 25 \mu \mathrm{m}$ in $\mathrm{x}$ and $\mathrm{y}$ from a line from the source to the center of the scintillator (line of sight 90-315, see Figure 2) used to detect the neutrons, pointing of the back of the pinhole to within $60 \mu$ radians of that line and placing the pinhole at a known distance from the source in the z-direction to within $\pm 250-\mu \mathrm{m}$. 
While these requirements have been temporarily loosened $( \pm 50 \mu \mathrm{m}$ in $\mathrm{x} \& \mathrm{y}$ and \pm 1 -mm in z) for the initial imaging campaigns, achieving alignment and holding alignment requires high stability of the diagnostic insertion module (DIM) on the 90-315 line-of-sight (LOS), the pinholes mounted on the 90-315 DIM, the target and the opposite port alignment system (OPAS) cameras on the 90-135 and 90-258 lines of sight.

As part of the NIS alignment commissioning, tests of the xy-alignment stability and angular stability of the pinhole array were included and, due to a shot delay, extended for a full twelve hours. In this report, we describe the stability tests and their results. Over a twelve-hour period, the relative $x-y$ stability of these parts was found to be within \pm 18 $\mu \mathrm{m}$ rms when using manual methods for tracking the position of the objects. (A portion of this range must be attributed to expected inconsistencies in any individual's ability to reproducibly determine feature locations within an image.) For highly visible features, use of basic particle tracking techniques found that the front of the pinhole array was stable relative to the $90-135$ OPAS camera to within $\pm 3.4 \mu \mathrm{m}$ rms. Video image recording of the specular reflection of an alignment laser (NILAD, please see section V for details) from a mirror mounted on the back of the pinhole array, and nearly perpendicular to the axis of the array, indicated angular stability better then 36 microradians. Reregistration, however, of the OPAS cameras themselves using the target alignment sensor (TAS) was found to change the expected position of target chamber center (TCC) by $194 \mu \mathrm{m}$. Thus while the NIS system was determined to be sufficiently stable relative to the OPAS cameras and the target, the potential position of the target placement could be far outside the tolerances for NIS. To deal with this problem, the 
alignment sequence was changed to include a final alignment of the NIS pinhole to the target after the final target positioning.

\section{NIS Alignment System}

Aligning the NIS pinhole array requires careful metrology of all of the components, as well as rough positioning and fine positioning of the assemblies. The initial rough positioning was achieved using a removable pointer that had been metrologized such that placing the 6-mm diameter ball at its' tip at TCC sets the initial z-position to within the $750-\mu \mathrm{m}$ pixel size of the CIVS cameras and places the pinhole array to within the travel range of the picomotor stack (Figure 3) that is used perform the fine alignment of the front and back of the pinhole. The fine alignment is achieved using the front minipenumbral pinholes and fiducials on the front and rear face of the pinhole, which are shown in Figures 4 and 5 as viewed in the NIS target chamber using the 90-135 OPAS.

\section{Initial Stability Measurements using the OPAS cameras}

\section{a. Setup}

To measure the stability of the system, the NIS DIM cart was placed at its rough alignment position with the front face of the pinhole at $32.5 \mathrm{~cm}$ from TCC with the attached blast shield opened so that assembly components would be visible. The CIVS lights were used to illuminate the front fiducials of the pinhole array and the OPAS 90135 illuminator was turned on to illuminate the rear fiducials of the pinhole array. The OPAS was registered to TCC using the TAS. The TCC registration pixel position on OPAS 90-135 was $(3932,2932)$. The 1.5-mm diameter exploding pusher target was then inserted near to TCC. 
In this study, we obtained images of the fiducials and mini-penumbral pinholes on the front of the pinhole array (Figure 4) and the fiducials at the back of the pinhole array (Figure 5). We also used the 90-135 OPAS camera to obtain images of the a bolt hold on the NIS blast shield mount that is used for rough alignment (Figure 6) and the 90-135 and 90-258 OPAS cameras to obtain images of the exploding pusher target (Figures 7 and 8, respectively). Eleven sets of these images were taken over $\sim 12$ hours between 19:55 on 2/16/11 and 9:30 AM on 2/17/11. In addition, CCD video video monitoring of the beam footprint from the specular reflection of an alignment laser (NILAD, please see section V for details) mounted within the NIS equipment annex was used to monitor angular pointing stability of the pinhole array. Bursts of 12 frames from the CCD video camera viewing the beam footprint on the alignment target were captured as part of the stability imaging sequences. A break in taking images occurred for a re-registration of the OPAS cameras and other target chamber activities between 1:30 AM and 5:30 AM on 2/17/11.

\section{b. Initial analysis}

The initial x-y analysis of the stability data was performed manually by placing circular regions of interest around the target features and finding the centroid of the circle. These results are shown in Table 1 for the stability of the center mini-penumbral pinhole (PHA Front) and the exploding pusher target on the 90-135 and 90-258 OPAS cameras. One pixel is $17 \mu \mathrm{m}$ at the target and $18 \mu \mathrm{m}$ at the front of the pinhole array. The standard deviations for all the data sets are between 0.4 and 1 pixel. These initial measurements show that the relative stability of the OPAS cameras, the target and the front of the pinhole array as mounted on DIM 90-315 was excellent, with relative motion less than 18 $\mu \mathrm{m}$ rms for the pinhole array and less than $17 \mu \mathrm{m}$ rms for the target capsule. 
Initial analysis of the angular stability as indicated by the alignment laser return consisted of visual observation of the video feed, in which only small motions of the return beam footprint were observed. (The return beam footprint in the annex is $\sim 6.6 \mathrm{~mm}$ in diameter, with manually observed motions of less than half of the footprint diameter.) Image analysis of the video frames of the alignment laser footprint indicated pinhole array angular stability of better than 36 micro-radians during the recorded period.

\section{c. OPAS 90-135 re-registration}

As mentioned above, the initial OPAS 90-135 target chamber center (TCC) registration position was $(3932,2932)$ pixels. A re-registration procedure was performed between measurements 6 and 7 and new TCC determined to be $(3943,2929)$ pixels. This represented an apparent TCC shift of $194 \mu \mathrm{m}$. Thus while the NIS system was stable relative to the OPAS cameras and the target, the potential position of the target placement could be far outside the tolerances for NIS. To deal with this problem, the NIS shot alignment sequence was changed to include a final alignment of the NIS pinhole to the target after the final target positioning.

\section{Additional analysis of the OPAS-based data sets}

While the results above are excellent, additional analysis of the data could be done on the front and rear fiducials of the pinhole array and the rough position of the DIM Cart, and we wanted to find more accurate and automated methods of analysis than simply placing a circle on the desired target and finding the centroid.

\section{a. Measurement of the front fiducial locations}

Because the mini-penumbral pinholes were visible at the front of the pinhole, which had not been a given when planning the experiment, they were used for the initial alignment 
in preference to the pre-designed front fiducials. The bead-blasting method used to produce the front fiducials on the black anodized surface of the fiducial plate, however, provides bright, high-contrast fiducials. The contrast of the fiducials makes it simple to apply techniques from particle tracking to observe the stability of the pinhole array. The method chosen here was to use ImageJ to make a binary image from the raw images, to define regions of interest for each fiducial and allow the program to determine the center of mass position of each fiducial for each measurement.

This method has the advantage that the center of mass of the binary image is virtually independent of the region of interest chosen for the measurement. In Figure 9a, we chose ten different regions of interest (rectangles, ellipses and freeform) on one of the binary images and measured the center of mass of the top left fiducial. The mean position of the center of mass for the ten measurements in pixels was $(3692.2017,2730.9074)$ with standard deviations of $(0.0031,0.0060)$ pixels. Since a pixel at this distance from the OPAS is approximately $18 \mu \mathrm{m}$, the standard deviations among the set of measurements are $(0.055 \mu \mathrm{m}, 0.107 \mu \mathrm{m})$, which allows accurate tracking of the position irrespective of the exact region of interest chosen.

Using the regions of interest shown in Figure 9b, we found that the mean $x-y$ positions in pixels of the centers-of-mass of the three fiducials over the course of the measurements were $(3692.1138,2730.8053),(4177.8719,2746.376)$ and $(3920.5004$, $3161.1761)$. The standard deviations of the measurements were $(0.175,0.156),(0.169$, $0.186)$ and $(0.183,0.156)$, respectively. Thus, the rms deviation was less than \pm 0.186 of a pixel in either $\mathrm{x}$ or $\mathrm{y}$ for all three fiducials, or less than $\pm 3.4 \mu \mathrm{m}$. This supports the results of the initial manual measurements that the stability of DIM relative to the 90-135 
OPAS was excellent and more than sufficient for holding the final alignment until the shot.

Since the three fiducials are hard mounted to the front of the pinhole, it is also expected that the motions of the pinhole should be highly correlated.

\section{b. Mini-penumbral pinhole positions}

The positions of the minipenumbral pinholes on the front of the pinhole body are visible on the front of the pinhole body as can be seen in Figure 4. We have analyzed the positions of the pinholes in the images in a manner similar to that for the front fiducials with the exception that instead of converting to binary images, we inverted the images and adjusted the minima and maxima of the images to obtain a similar effect in the local region around the minipenumbral pinholes. Regions of interest were again defined and the center of mass for each image was obtained. The mean position and standard deviations were then obtained for each pinhole. The mean $(\mathrm{x}, \mathrm{y})$ positions were (3906.0796, 2878.7865), $(3925.34,2878.7344)$ and $(3946.9299,2880.5065)$ pixels for the left, center and right pinholes, respectively. The $(\mathrm{x}, \mathrm{y})$ standard deviations were $(0.160$, $0.146),(0.187,0.163)$ and $(0.181,0.167)$, respectively. We note that the left pinhole was less well defined and may have been influenced by signal at the edge of the region of interest. Overall, the standard deviations of the positions for the minipenumbrals are comparable to those for the front fiducials, and the maximum deviation again corresponds to $\sim 3.4 \mu \mathrm{m}$.

We note that the data sets for the front fiducials and the minipenumbral pinholes essentially constitute a characterization of the locations of the fiducials relative to the pinholes. While the apparent positions might change slightly due to changes in lighting, 
the lighting levels in the chamber were adjusted extensively during the course of the experiment, which is apparent from images of the exploding pusher target in particular, and as long as the contrast is maintained, changes in position due to lighting appear to be minimized by the flatness of the front face of the pinhole.

\section{c. Rear fiducial positions}

While purely manual measurement of the position of the fiducials at the rear of the pinhole (Figure 5) is possible and yields similar \pm 1 pixel results to all the other manual measurements in this report, more automated measurement of the rear fiducials proved to be significantly more difficult than measuring those at the front due to lower contrast and poorer lighting conditions, including multiple reflections of the fiducials in the center of the image that frequently actually overlap the primary observed fiducial locations. The latter effect is visible on the lower right fiducial in Figure 5b. For future attempts to automate the measurement of the rear fiducials, a procedure to set and control the lighting will be required. Investigation of the means to reduce the reflections in the center of the images may also prove useful.

\section{d. Target position using 90-135 OPAS and 90-258 OPAS}

While we did attempt to automate the measurement of the target position from the 90-135 and 90-258 OPAS images, the inherent low contrast and the significant changes in the bright spots that occurred due to changes in the chamber illumination prevented any particular success. The results were similar to the manual measurements discussed in Section III. It is possible that additional image processing or careful control of the chamber lighting would allow more accurate measurements in the future, but it was 
determined that at this point no additional attempts at either data analysis or obtaining a better data set were warranted.

\section{e. Rough alignment bolt hole fiducial position}

Because the bolt-hole fiducial (Figure 6b) that is used for rough alignment is not designed to be insensitive to illumination conditions, we found that the position was quite sensitive to the chamber illumination. Tracking the position by inverting the image and making it binary, we found that the mean $(\mathrm{x}, \mathrm{y})$ position was $(6033.62,3356.52)$ pixels with standard deviations of $(8.3,13.2)$ pixels. At this position a pixel is $\sim 17 \mu \mathrm{m}$, so this represents deviations of $(140.5 \mu \mathrm{m}, 224.6 \mu \mathrm{m})$, which is sufficient for rough alignment but inadequate for fine system alignment, which is required for data collection. We note, however, that if two images with unusually low lighting are removed, the mean position changes to $(6037.5,3362.56)$ pixels with standard deviations of $(0.33,0.85)$ pixels, and more accurate measurements could be made.

\section{Pointing stability measurements using NILAD}

The low-power, eye-safe 532-nm laser system known as the NILAD, which is mounted in the neutron-imaging annex, allowed another measurement of the stability of the pointing of the pinhole towards the scintillator array. The laser is pointed from the annex and reflected from a mirror mounted on the back of the pinhole array assembly. Since the mirror is aligned nearly normal to the axis of the pinhole array, the beam reflects back to the annex where it can be seen on a screen using a CCD camera as shown in Figure 12.

For the measurement, we obtained fifteen sets of twelve images over the same twelve hours during which we made the previous measurements. We analyzed the images by performing a background subtraction based on all the images to remove the grid from 
the images. Making binaries of the images was not required since the contrast after background subtraction was excellent. A circular region of interest encompassing all the spot positions was set, and ImageJ was used to automatically calculate the center of mass for all the images. The standard deviation of the mean position of the spots was $0.20 \mathrm{~mm}$ in $\mathrm{x}$ and $0.20 \mathrm{~mm}$ in $\mathrm{y}$. At this position, this corresponds to $7.8 \mu$ radians and $7.8 \mu$ radians in $\mathrm{x}$ and $\mathrm{y}$, respectively. Using the $3.4 \mu \mathrm{m}$ stability of the pinhole found in Section IVa for both front and back of the pinhole and assuming that the deviations are in opposite directions for the front and back of the array, which is an extreme assumption, would indicate that the maximum angular deviation should be less than $36 \mu$ radians rms in $\mathrm{x}$ and $\mathrm{y}$, so this result is consistent with the observed stability using the OPAS to monitor the pinhole. We note that the maximum observed radial deviation from the mean position was $1.01 \mathrm{~mm}$, which corresponds to $39 \mu$ radians.

\section{Conclusions}

The alignment system for the National Ignition Facility’s neutron imaging system has been commissioned and measurements of the relative stability of the 90-315 DIM, the front and the back of the neutron imaging pinhole array, and an exploding pusher target have been made using the $90-135$ and the $90-258$ opposite port alignment systems. Over a twelve hour period, the relative stability of these parts was found to be excellent, within $\sim \pm 18 \mu \mathrm{m}$ rms, even when using manual methods for tracking the position of the objects.

For highly visible features, such as the pinhole front fiducials and the mini-penumbral pinholes, use of basic particle tracking techniques found that the front of the pinhole array was stable relative to the $90-135$ opposite port alignment camera to within $\pm 3.4 \mu \mathrm{m}$ rms. Reregistration, however, of the opposite port alignment systems themselves using 
the target alignment sensor was found to change the expected position of target chamber center by up to $194 \mu \mathrm{m}$.

For future alignment of the NIS system, it will be necessary to align to the finally aligned target position until the OPAS registration position issue is understood. Other possible improvements include improved lighting and consequent observed contrast for the rear PHA fiducials and more careful control of lighting.

\section{References}

1. G. P. Grim, C. W. Barnes, P. A. Bradley, et al., Journal De Physique Iv 133, 913 (2006).

2. V. Y. Glebov, D. D. Meyerhofer, T. C. Sangster, et al., Review Of Scientific Instruments 77, 10E715 (2006).

3. M. D. Wilke, S. H. Batha, P. A. Bradley, et al., Review of Scientific Instruments 79, 10E529 (5 pp.) (2008).

4. G. P. Grim, P. A. Bradley, R. D. Day, et al., Journal of Physics: Conference Series 112, 032078 (4 pp.) (2008).

5. D. C. Wilson, G. P. Grim, I. L. Tregillis, et al., Review Of Scientific Instruments 81 (2010). 


\section{Figure Captions}

Figure 1 Image of the rear surface of the neutron imaging pinhole array. The image shows a row of three circular mini-penumbral pinholes in the center and two rows each of triangular pinholes above and below. For scale, the triangular pinholes are $226-\mu \mathrm{m}$ and the mini-penumbral pinholes are $437-\mu \mathrm{m}$ in diameter at the rear surface of the pinhole array.

Figure 2 Schematic of the NIS installed on LOS 90-315. The NIS LOS runs from TCC through the pinhole array, which is held on the DIM cart, and the collimators in the target bay and switchyard walls and in to the NIS Annex where the scintillator and cameras are placed.

Figure 3 Vertical cut through a model of the NIS snout. For alignment, the blast shield rotates out of the line of sight. The pinhole array is mounted to a picomotor driven stage that allows micron-level control of the $x-y$ position of the front and the pitch and yaw of the pinhole body.

Figure 4 NIS pinhole alignment fiducials at the front and back of the pinhole array as viewed in the NIS target chamber using the 90-135 OPAS. a) The full image of the NIS pinhole alignment fiducials at the front of the pinhole array. The large circular opening is the front of the NIS shroud. The three small bright spots in a triangle pattern near the center of the image are the alignment fiducials. b) A zoomed view of the front of the pinhole array. The three small black dots in a 
horizontal row at the center are the mini-penumbral pinholes, and the three larger bright spots (bottom center and upper right and left) are the 2-mm diameter beadblasted fiducial marks.

Figure 5 NIS pinhole alignment fiducials at the back of the pinhole array as viewed in the NIS target chamber using the 90-135 OPAS. a) Full image focused on the rear alignment fiducials at the rear of the pinhole. b) Zoomed image of the NIS pinhole alignment fiducials at the rear of the pinhole array. The rear three dark holes in the aluminum tabs (top center and bottom right and left) are the 2-mm diameter fiducials.

Figure 6 OPAS 90-135 image of the bolt hole used as a rough alignment feature. a) Full image focused on plane of the bolt hole, which is shown just below the three o'clock position on the outside of the NIS shroud pipe. It is part of the assembly that holds the blast shield. b) Zoomed image of the image of the bolt hole used as a rough alignment feature.

Figure 7 OPAS 90-135 image of the 1.5-mm exploding pusher target. a) Full image b) Zoomed image of the target. The bright spots on the sphere of the target are due to the illumination for the CIVS cameras. 
Figure 8 OPAS 90-258 image of the 1.5-mm exploding pusher target. a) Full image b) Zoomed image of the target. The bright spots on the sphere of the target are due to the illumination for the CIVS cameras.

Figure 9 Example conversion of an image of the front fiducials to a binary image. a) Image with ten different regions of interest for a single fiducial in blue. b) Image showing the three regions on interest used to track the position of the front fiducials.

Figure 10 Plots of the positions of the top two front fiducials on the PHA versus the position of the bottom center fiducial. The plots show the expected correlation between the positions of the three fiducials. All positions given are relative to the mean positions. a) Plot in the $x$ dimension, b) Plot in the $y$ position

Figure 11 Plots of the positions of the left and right minipenumbral pinhole on the front of the PHA versus the position of the center minipenumbral pinhole. The plots show the expected correlation between the positions of the three pinholes. All positions given are relative to the mean positions. a) Plot in the $x$ dimension, b) Plot in the $y$ position

Figure 12 Image of the NILAD laser beam on the target screen in the NIS Annex. The beam exits the screen through the hole at the upper right, travels to the mirror mounted at the back of the pinhole array in the 90-315 DIM and reflects back to the screen. The squares on the screen are $1-\mathrm{cm}$ square. 
LLNL-xxxx-xxxxxx

\section{Figures}

Figure 1

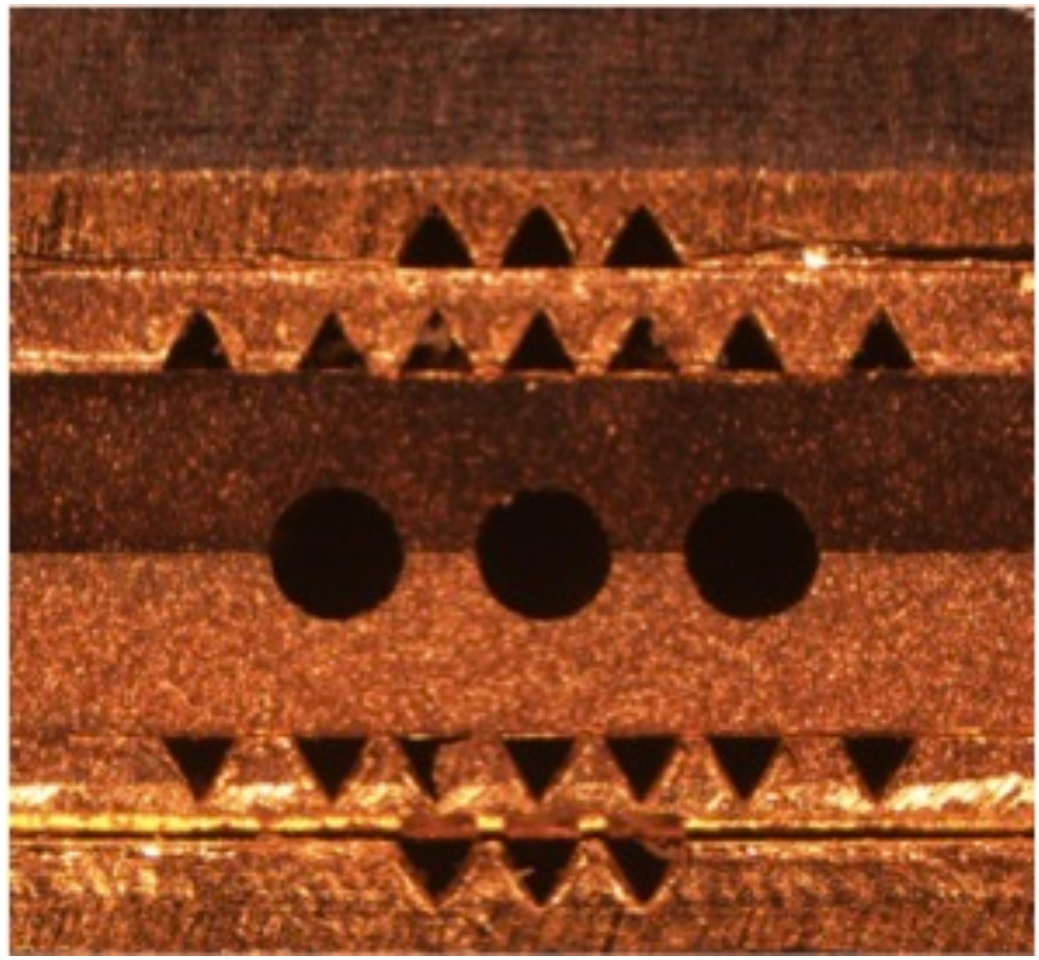


Figure 2

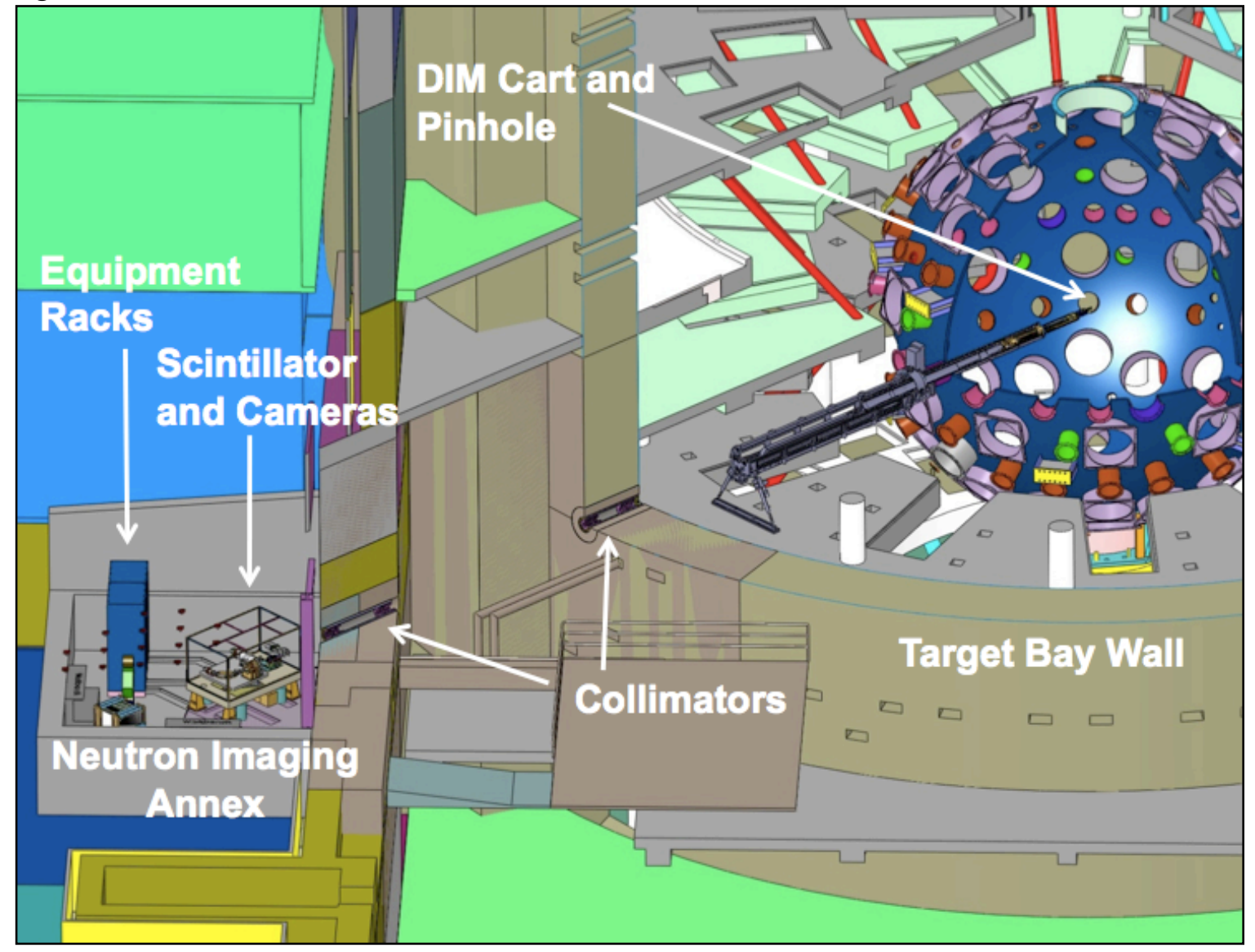


Figure 3

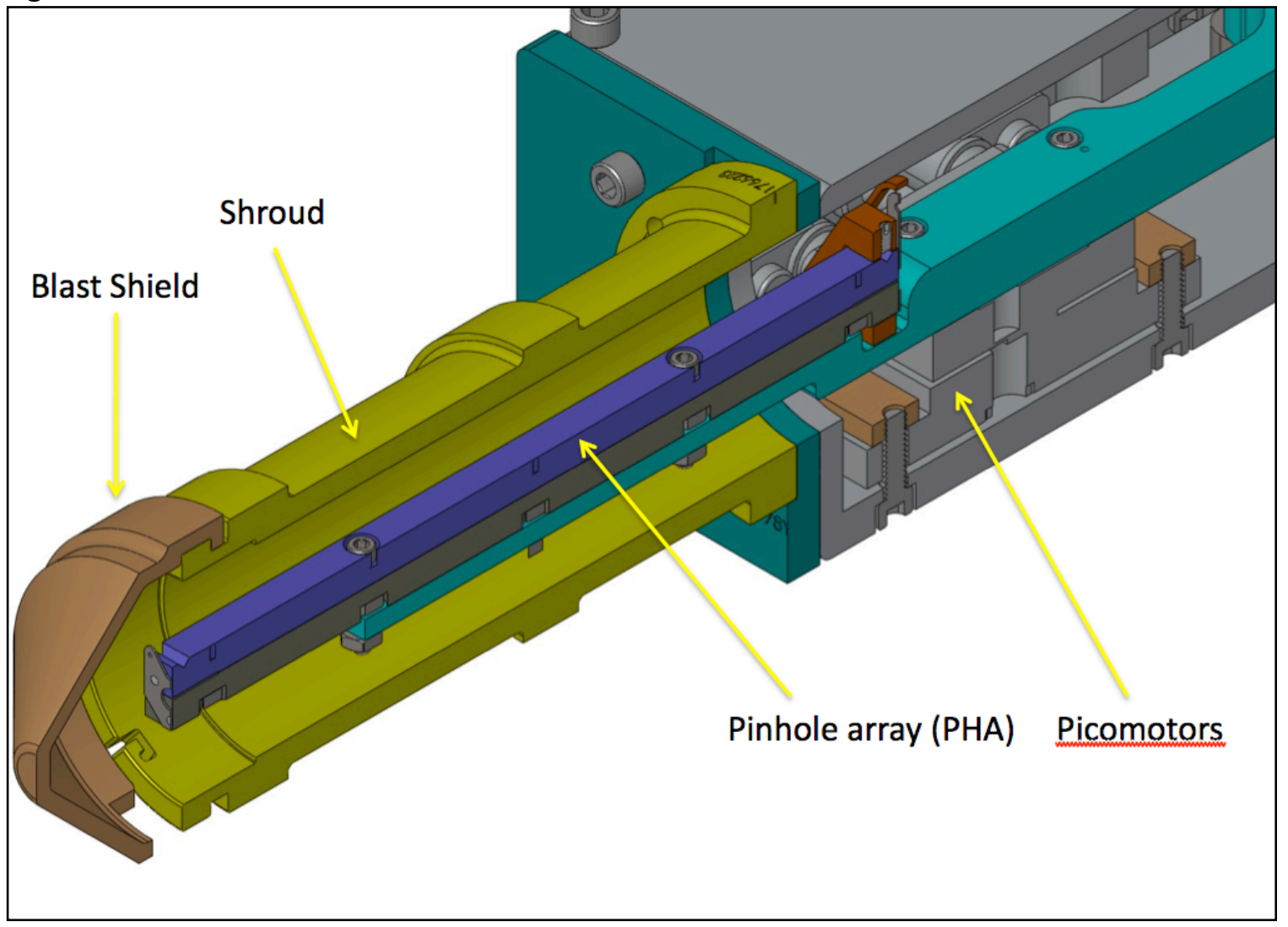


Figure 4a

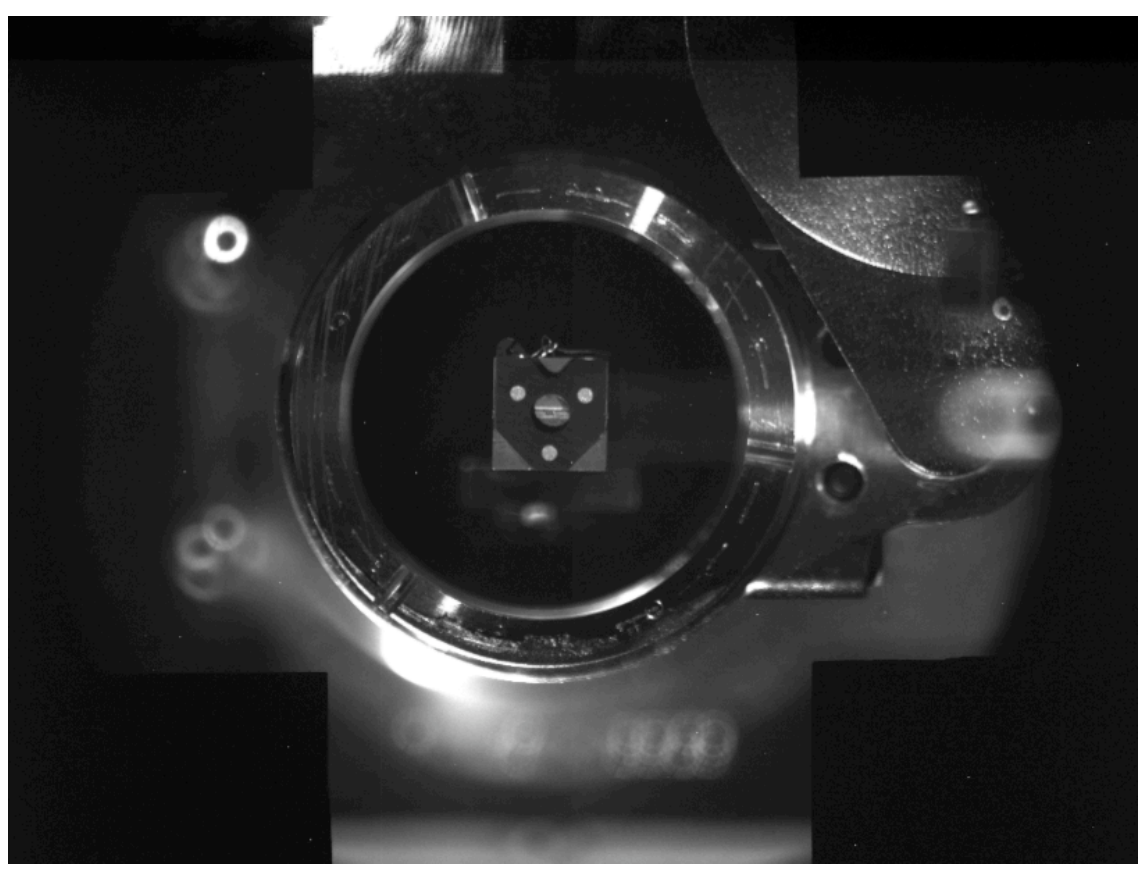

Figure 4b

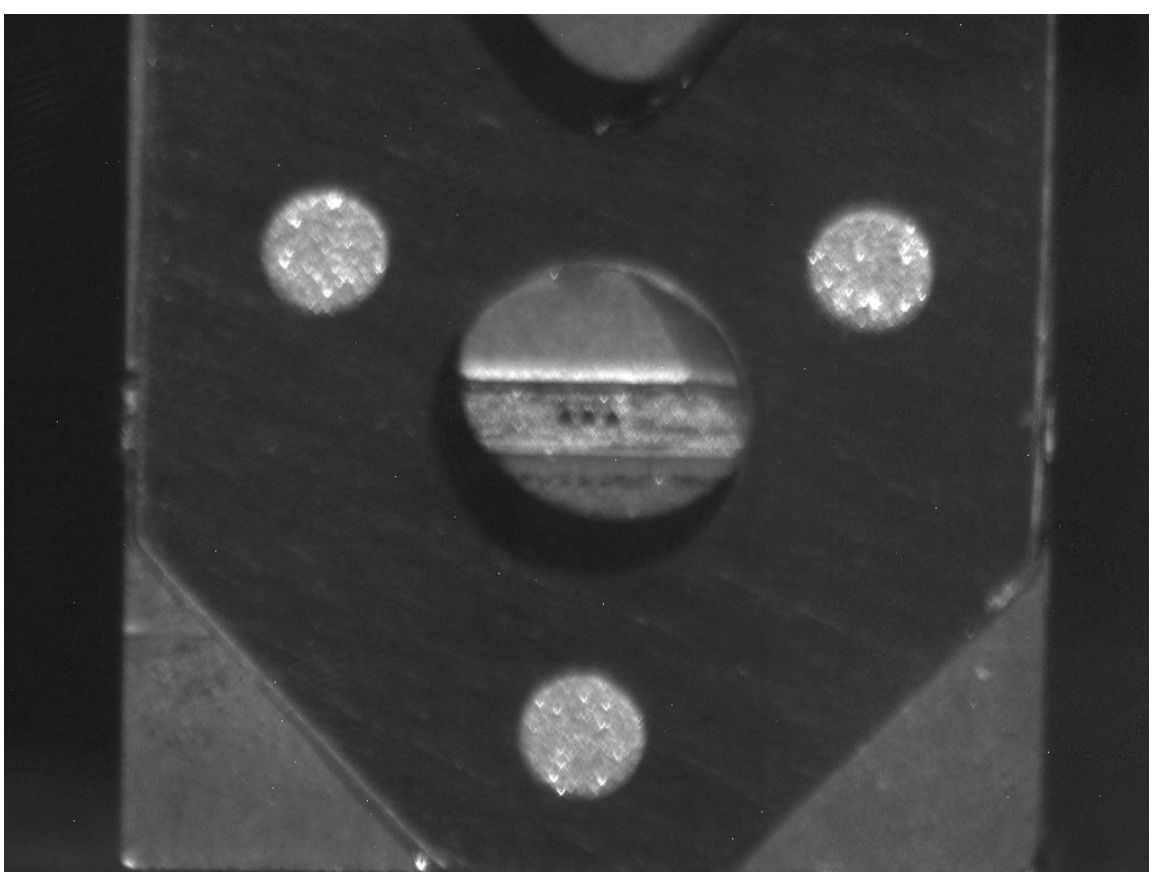


Figure 5a

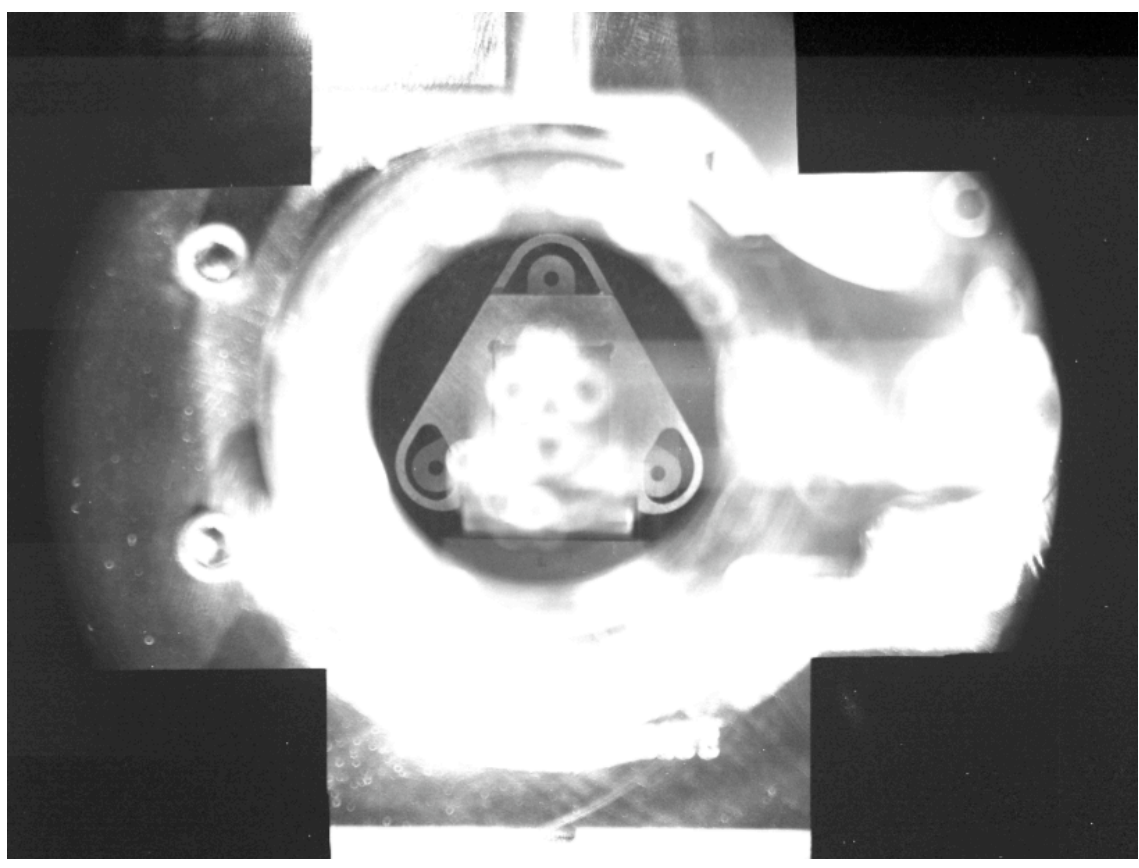

Figure 5b

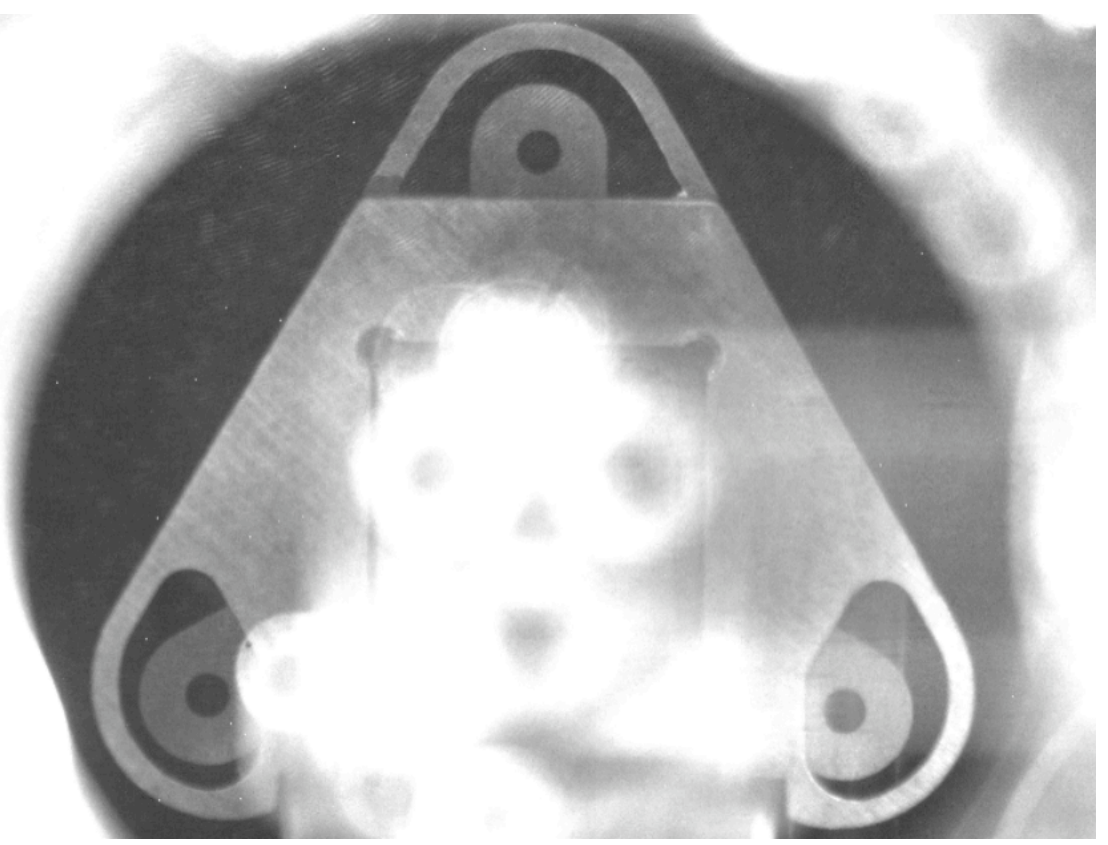


Figure 6a

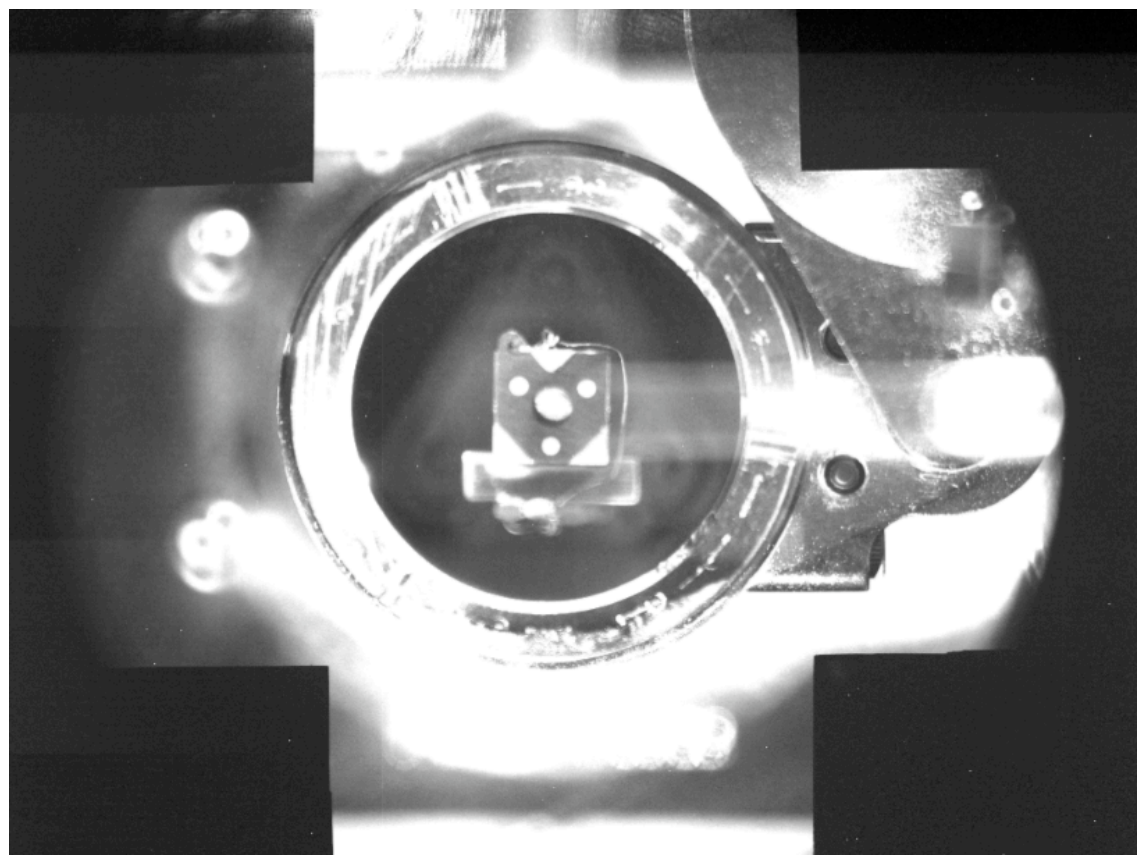

Figure $6 b$

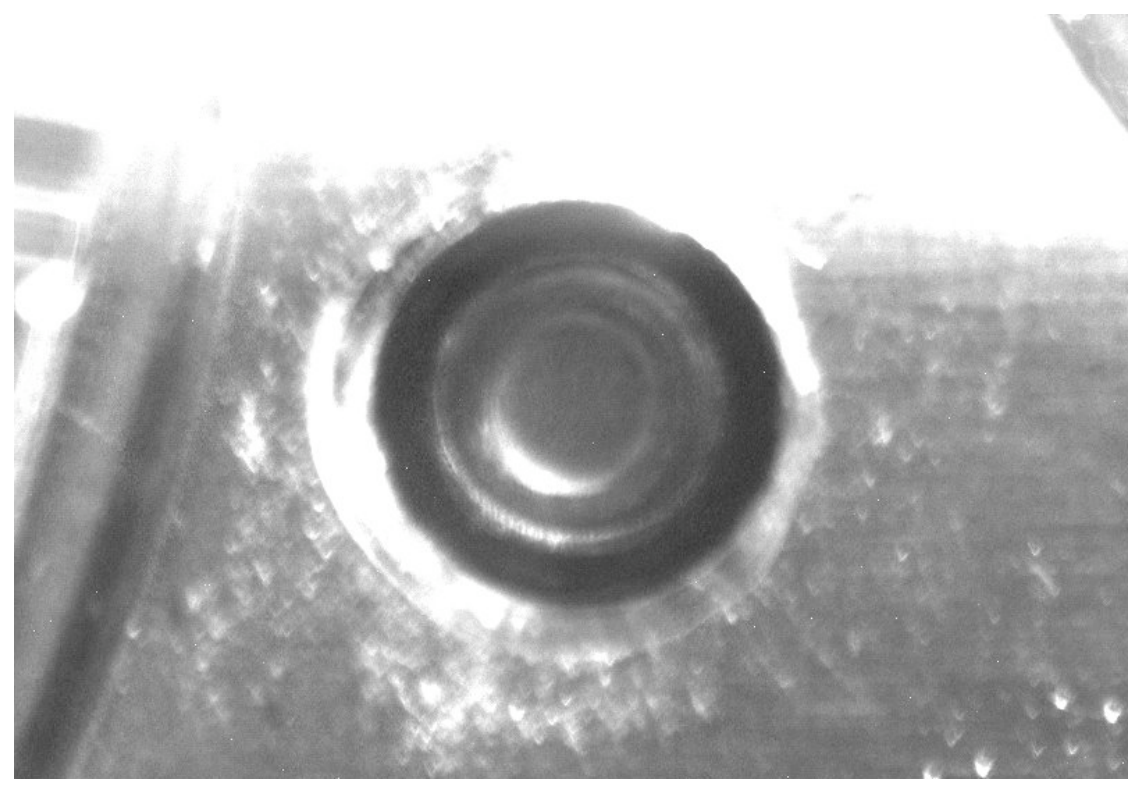


Figure $7 \mathrm{a}$

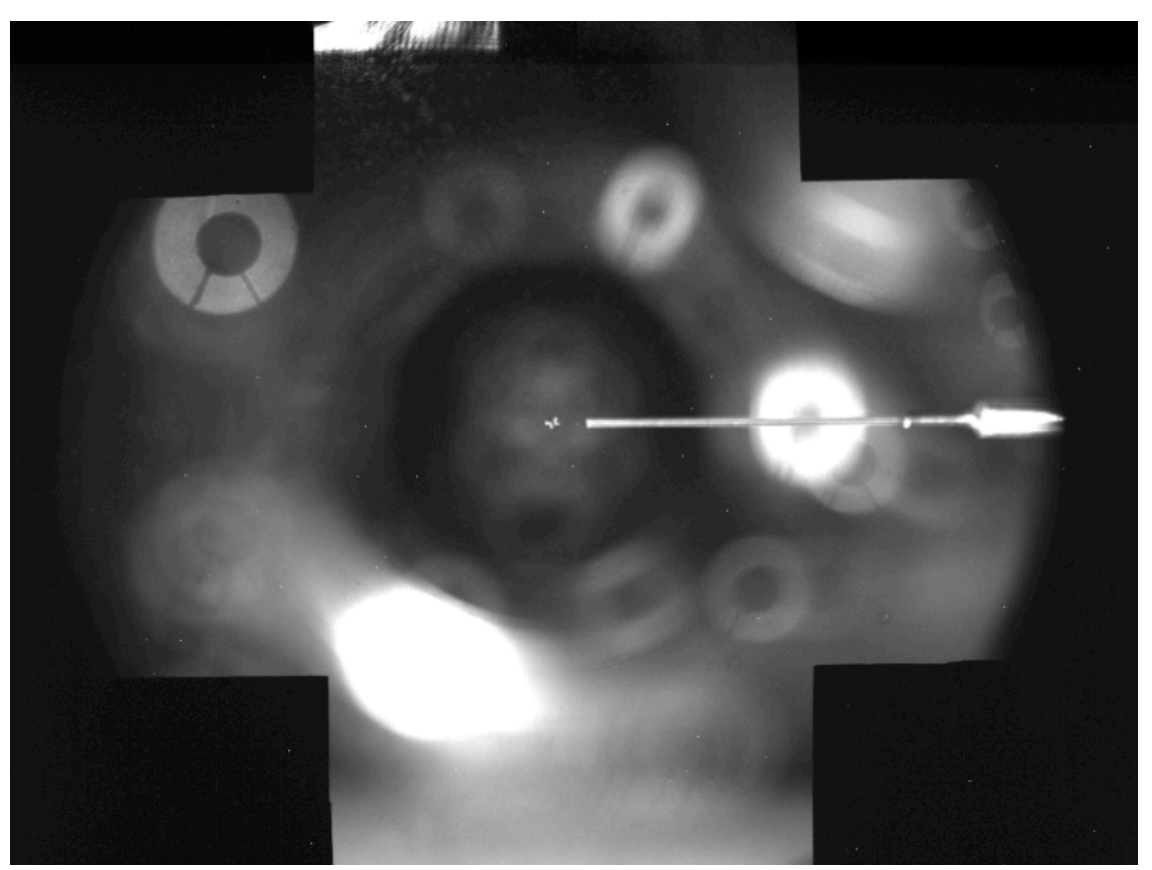

Figure $7 b$

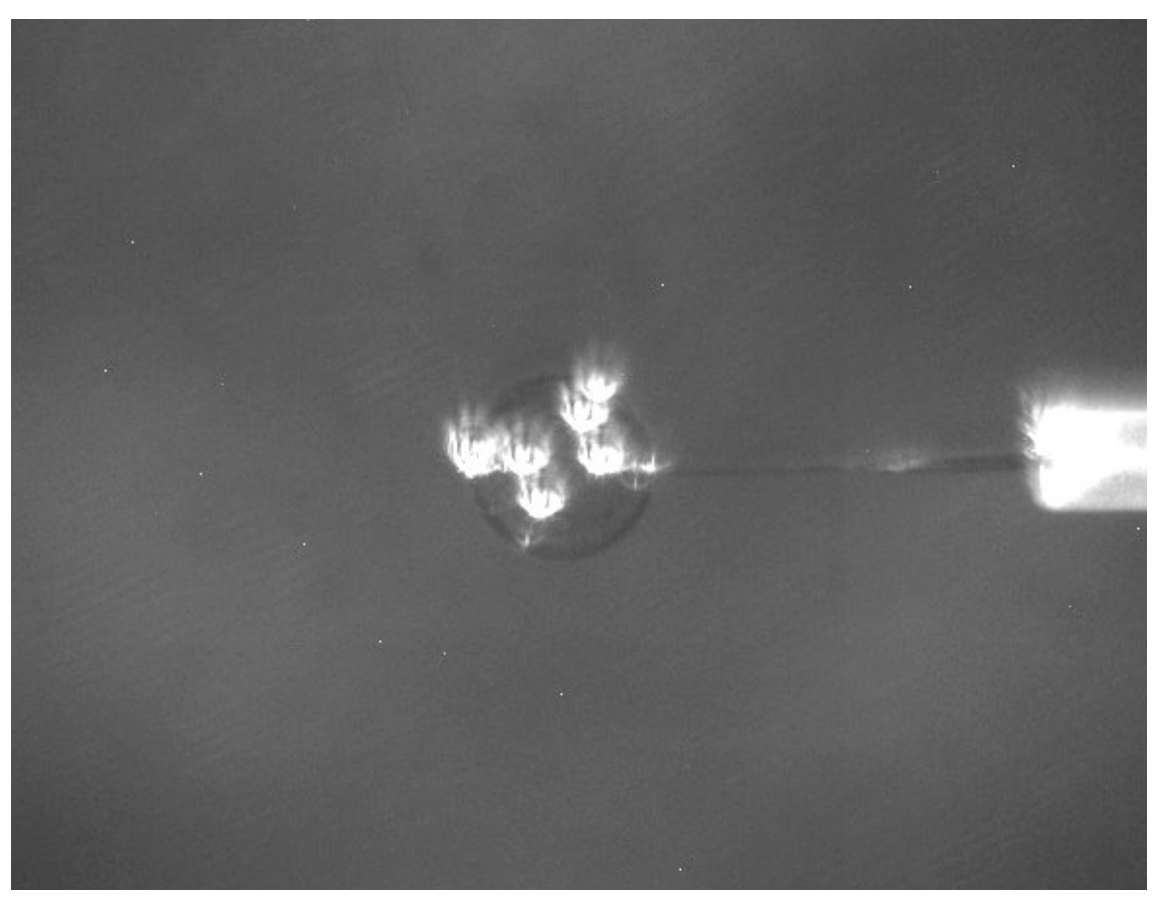


Figure 8a

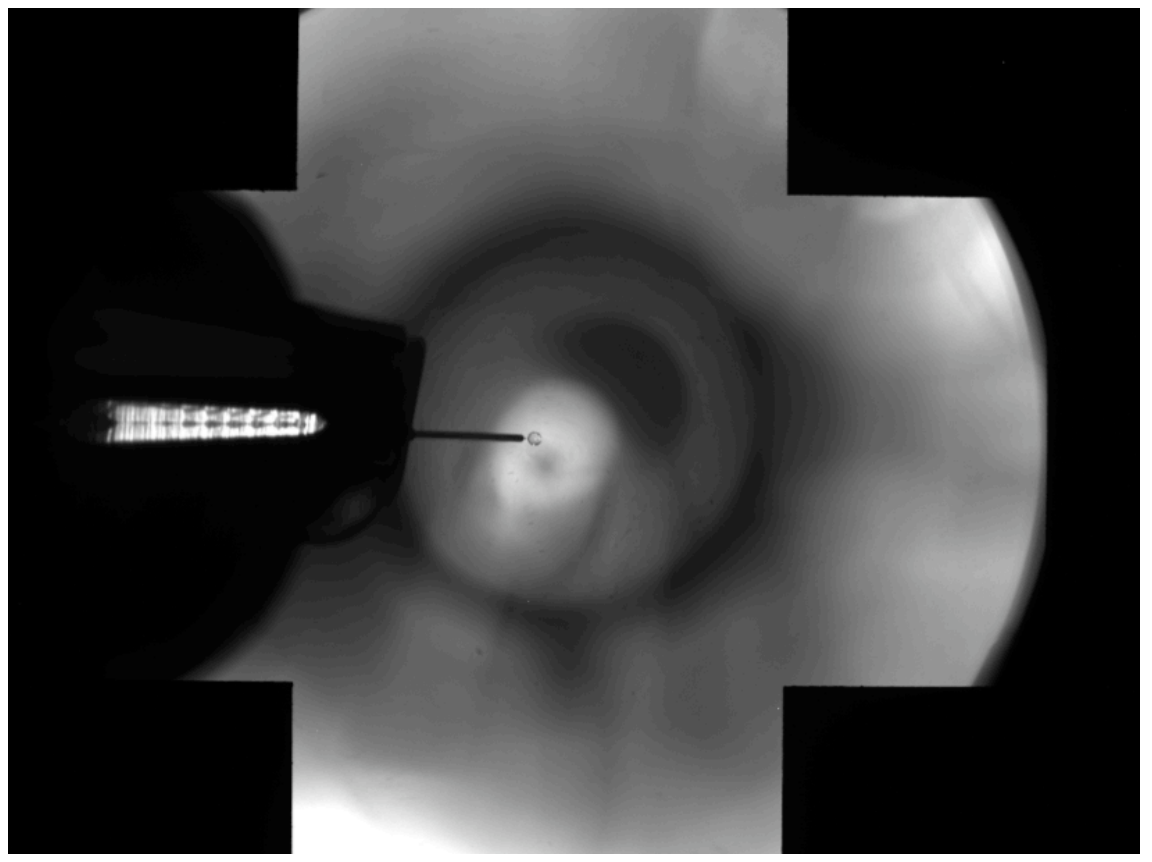

Figure $8 b$

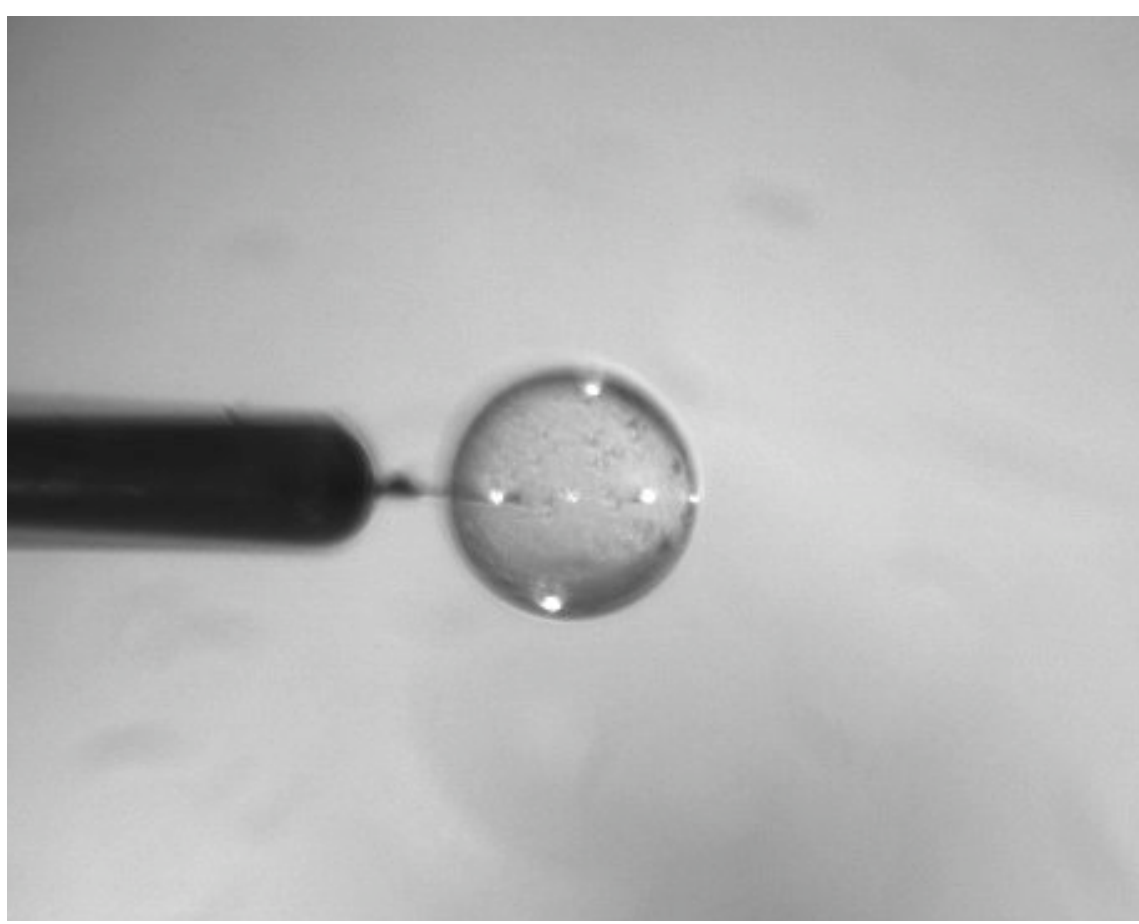


Figure 9a

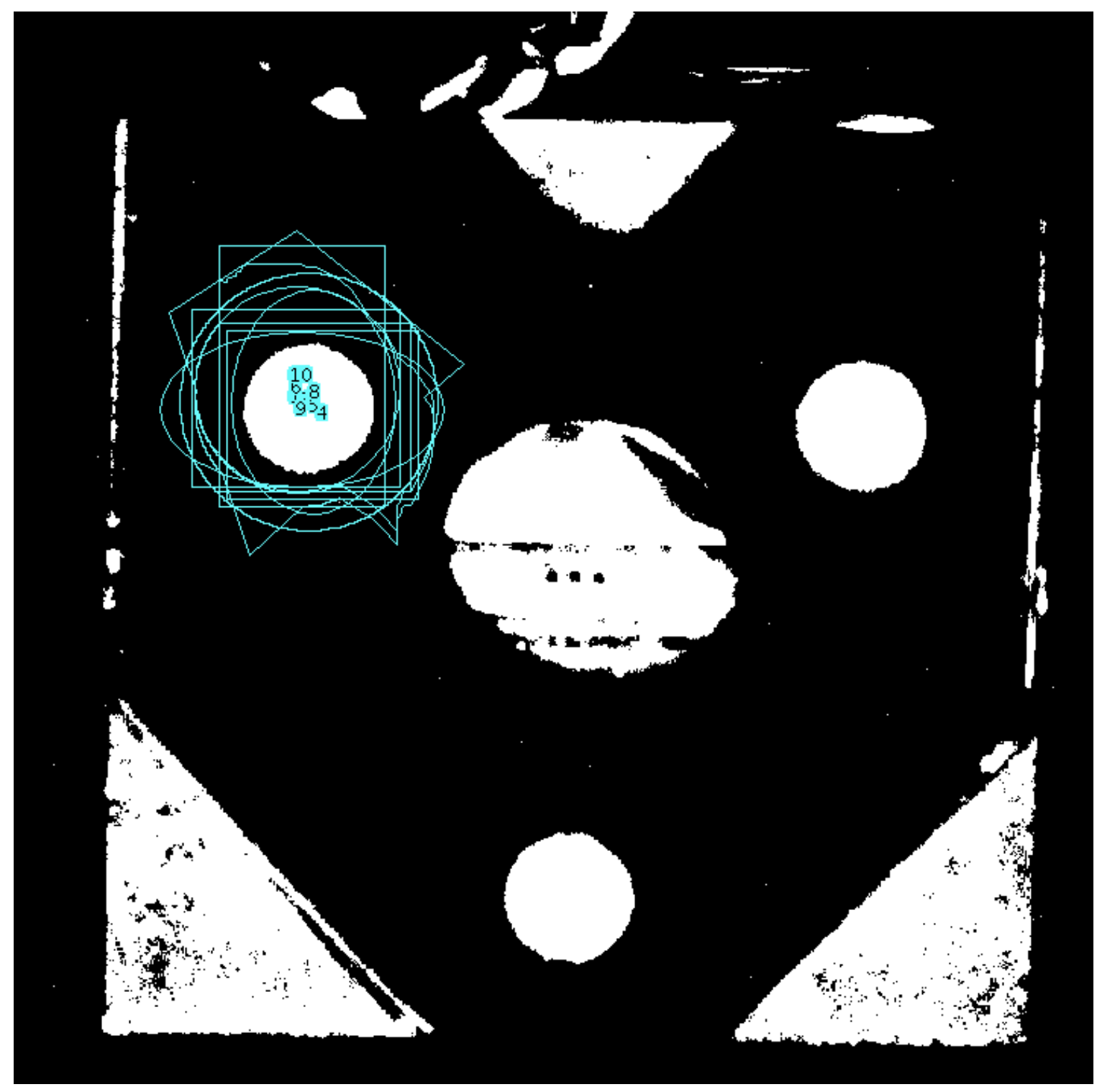


Figure $9 b$

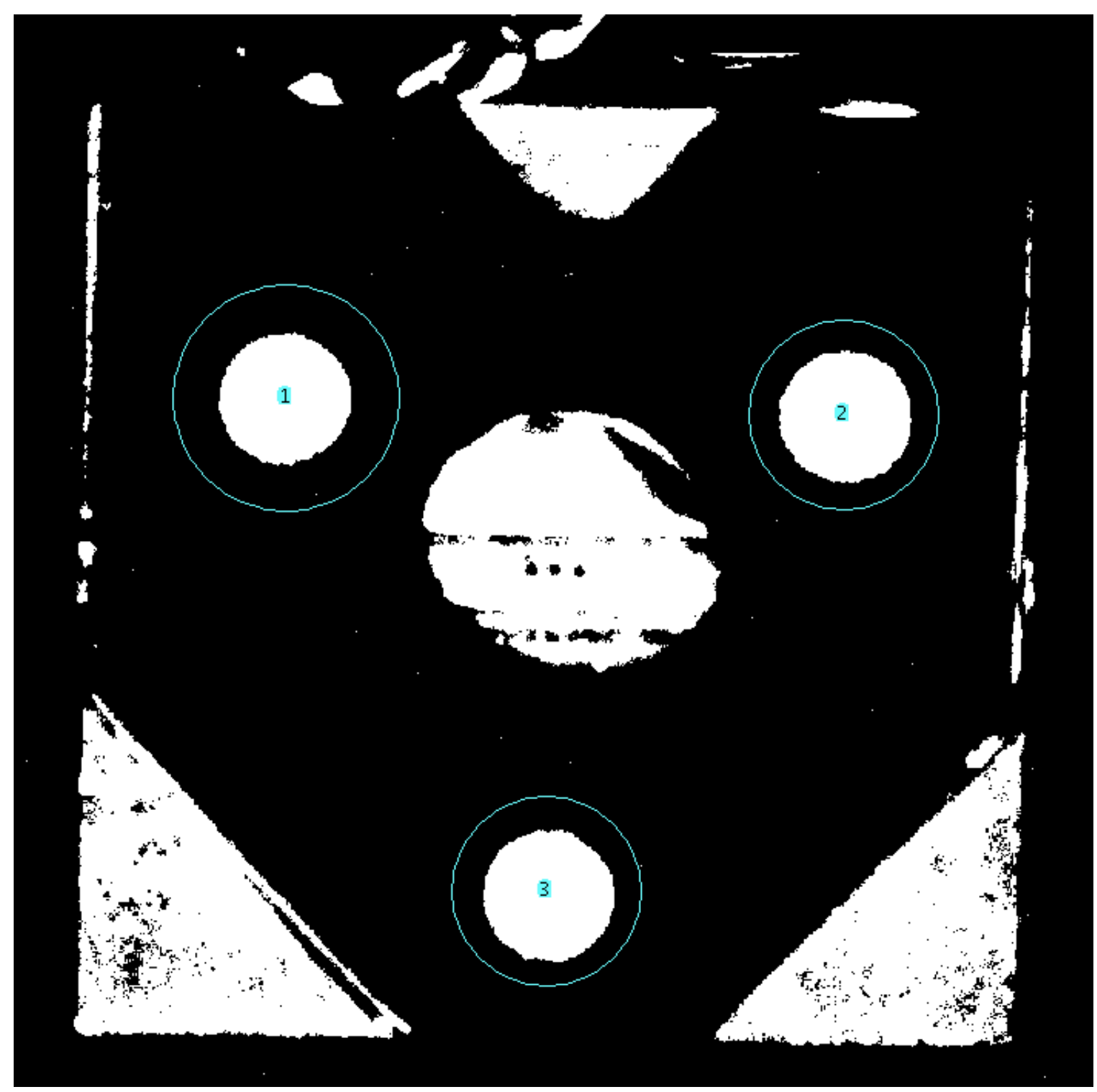


Figure 10
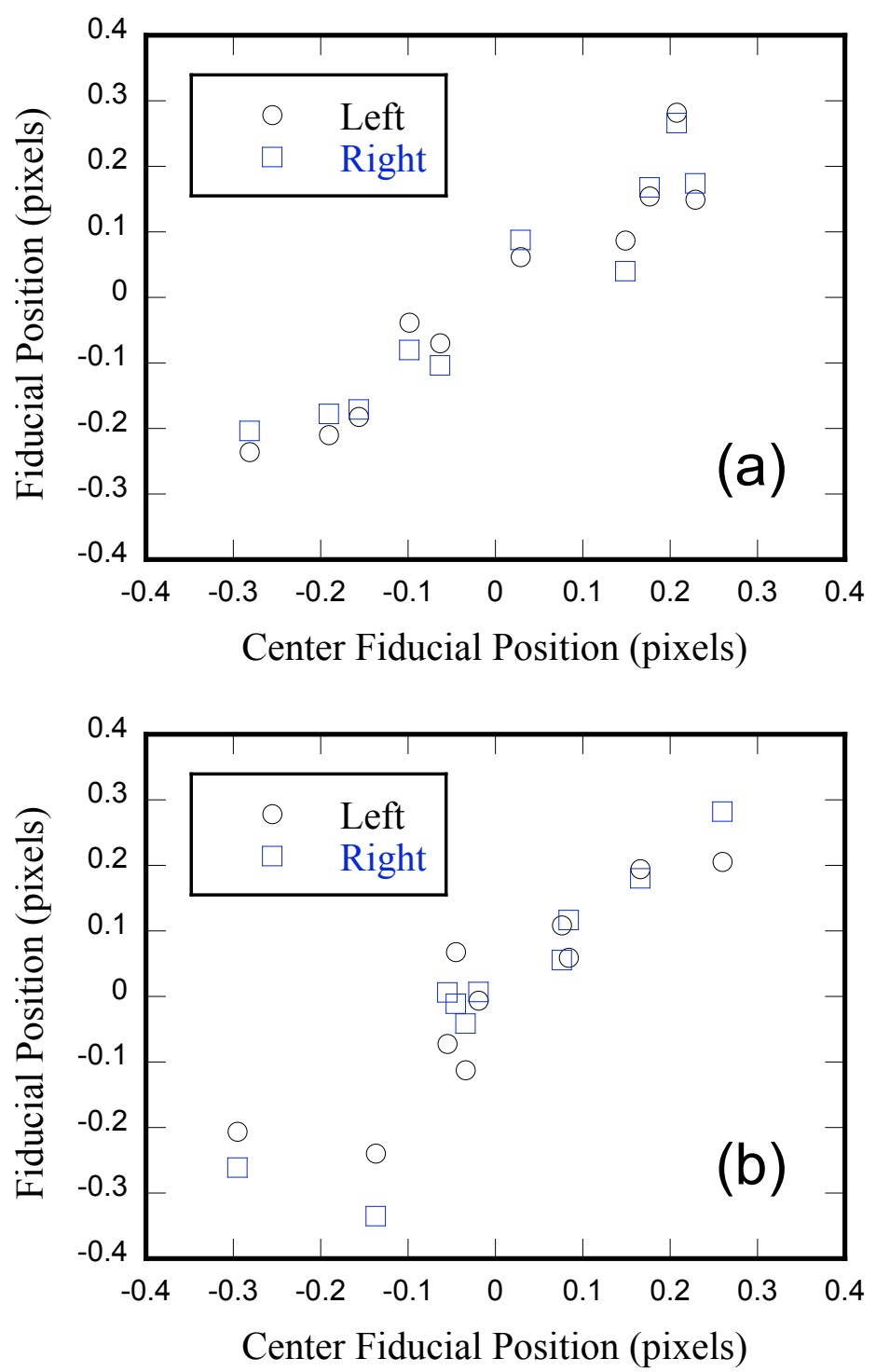
Figure 11
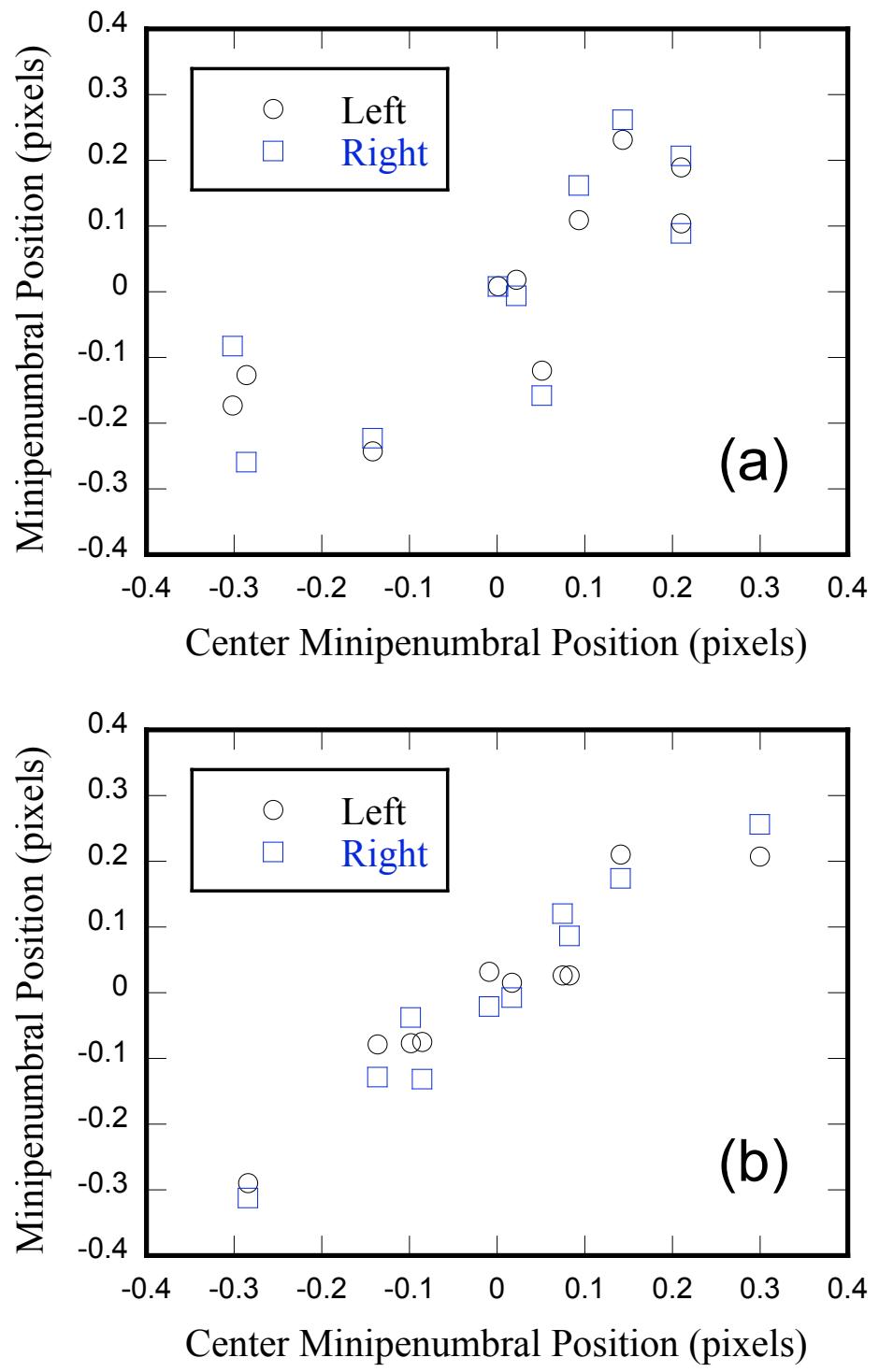
Figure 12

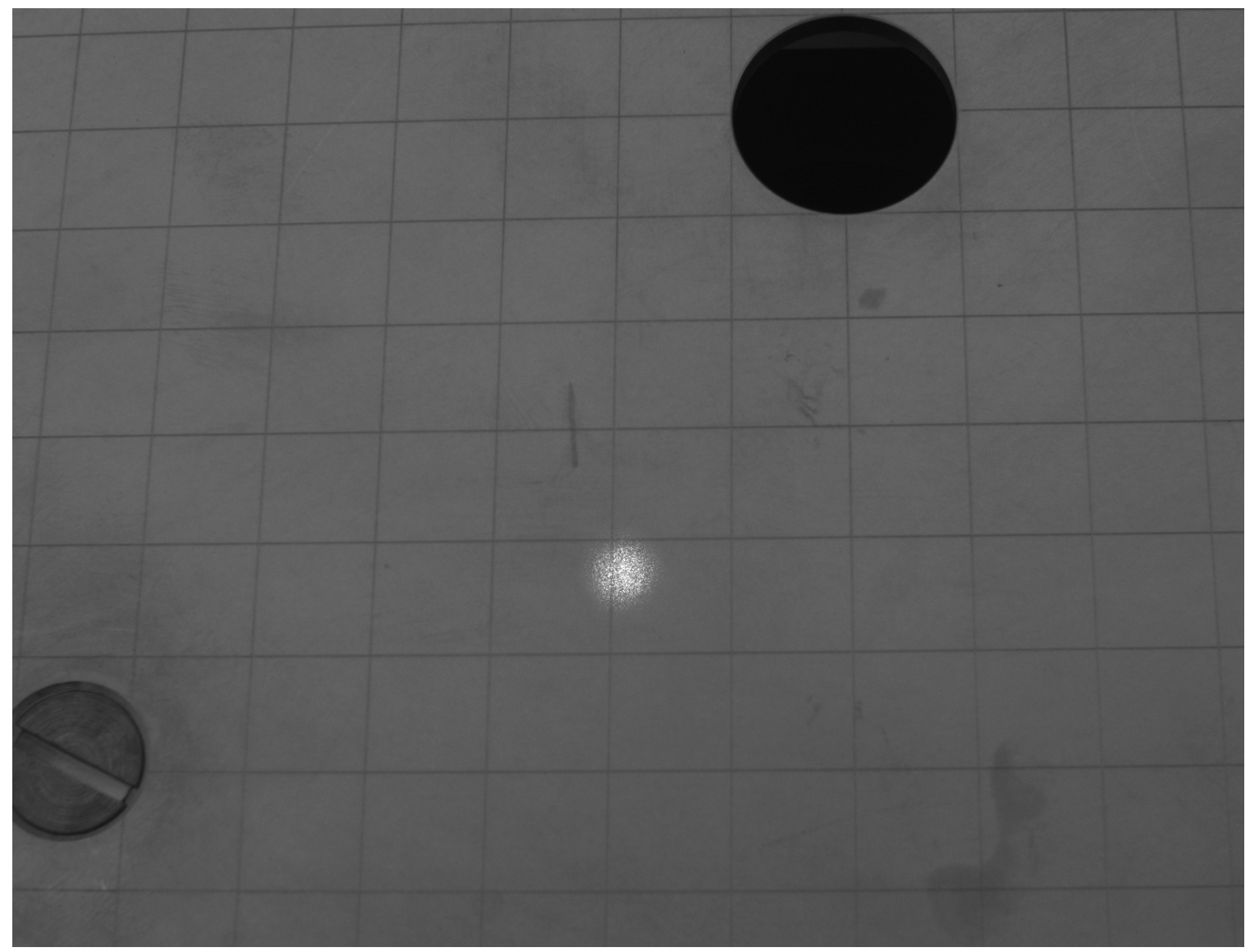




\section{Tables}

Table 1

\begin{tabular}{|c|ccccccc|}
\hline Measurement & Time & \multicolumn{2}{c}{ PHA Front } & \multicolumn{2}{c|}{ Target } & 90-135 & \multicolumn{2}{c|}{ Target } & 90-258 \\
\hline & & $\begin{array}{c}\mathrm{x} \\
\text { (pixels) }\end{array}$ & $\begin{array}{c}\mathrm{y} \\
\text { (pixels) }\end{array}$ & $\begin{array}{c}\mathrm{x} \\
\text { (pixels) }\end{array}$ & $\begin{array}{c}\mathrm{y} \\
\text { (pixels) }\end{array}$ & $\begin{array}{c}\mathrm{x} \\
\text { (pixels) }\end{array}$ & $\begin{array}{c}\mathrm{y} \\
\text { (pixels) }\end{array}$ \\
1 & $20: 46$ & 3925 & 2879 & 3939 & 2929 & & \\
2 & $21: 34$ & 3925 & 2879.5 & 3938.5 & 2929 & 3808 & 3124 \\
3 & $22: 32$ & 3925.5 & 2879.5 & 3939.5 & 2929.5 & 3807.5 & 3122.5 \\
4 & $23: 29$ & 3926 & 2879 & 3937.5 & 2929 & 3808 & 3122 \\
5 & $0: 34$ & 3925 & 2879.5 & 3940 & 2928 & 3807.5 & 3122 \\
6 & $1: 16$ & 3925.5 & 2879.5 & 3940 & 2928 & 3807.5 & 3123 \\
7 & $5: 30$ & 3925.5 & 2878.5 & 3939 & 2926.5 & 3806 & 3124 \\
8 & $6: 00$ & 3925.5 & 2879.5 & 3940 & 2927 & 3806 & 3123 \\
9 & $7: 47$ & 3925.5 & 2879.5 & 3939 & 2929 & 3806 & 3123 \\
10 & $8: 33$ & 3925 & 2880 & 3939 & 2929 & & \\
11 & $9: 30$ & 3925 & 2880 & & & & \\
\hline
\end{tabular}

\section{Table Captions}

Table 1 Initial manual measurements of the stability of the center mini-penumbral pinhole (PHA Front) and the exploding pusher target on the 90-135 and 90-258 OPAS cameras. One pixel is $17 \mu \mathrm{m}$ at the target and $18 \mu \mathrm{m}$ at the front of the pinhole array. The standard deviations for all the data sets are less than 1 pixel, which is probably set by the ability of the operator to choose region of interest for the measurement.

\section{Disclaimer}

This document was prepared as an account of work sponsored by an agency of the United States government. Neither the United States government nor Lawrence Livermore National Security, LLC, nor any of their employees makes any warranty, expressed or implied, or assumes any legal liability or responsibility for the accuracy, completeness, or 
usefulness of any information, apparatus, product, or process disclosed, or represents that its use would not infringe privately owned rights. Reference herein to any specific commercial product, process, or service by trade name, trademark, manufacturer, or otherwise does not necessarily constitute or imply its endorsement, recommendation, or favoring by the United States government or Lawrence Livermore National Security, LLC. The views and opinions of authors expressed herein do not necessarily state or reflect those of the United States government or Lawrence Livermore National Security, LLC, and shall not be used for advertising or product endorsement purposes. 